\title{
The Hopf Ring for Morava $\mathbb{K}$-Theory
}

\author{
By \\ W. Stephen WILSON*
}

\section{Introduction}

Morava $K$-theories were first introduced and studied by Jack Morava. For $p$ a prime, and $n>0, K(n)_{*}(-)$ is a periodic generalized homology theory of period $2\left(p^{n}-1\right)$. The coefficient ring is $K(n)_{*} \cong Z / p\left[v_{n}, v_{n}^{-1}\right],\left|v_{n}\right|=2\left(p^{n}-1\right)$. For $n=1, K(1)_{*}(-)$ is a summand of the mod $p$ complex $K$-theory homology. For higher $n$, Morava proved a generalized Conner-Floyd theorem which led to a solution of the Conner-Floyd conjecture, see $[\mathbb{J}-\mathbb{W}]$ and $\left[\mathbb{R}-\mathbb{W}_{2}\right]$. Morava also developed the properties of the stable operations. This was instrumental in the study of stable homotopy in [MRW]. Morava has several unpublished papers on his work, for example, $\left[\mathbb{M}_{1}\right]$ and $\left[\mathbb{M}_{2}\right]$. The purpose of this paper is to describe the unstable Morava $K$-theory operations.

Let $K(n)_{*}=\left\{K(n)_{i}\right\}$ be the $\Omega$-spectrum representing the $n$-th Morava $K$-theory for a fixed prime $p$. Because this is periodic, i.e., $K(n)_{i} \simeq K(n)_{i+2\left(p^{n}-1\right)}$, we consider it as graded over $Z / 2\left(p^{n}-1\right)$ instead of $Z$. Our goal is to compute $K(n)_{*} K(n)_{*}$, which is dual to the unstable operations. We actually compute $E_{*} \underline{K(n)} *$ for a wide class of generalized homology theories $E_{*}(-)$. Let $E$ be a multiplicative $B P$ module spectrum, i.e., a ring spectrum with a multiplicative map $\mu: B P \rightarrow E$.

Let $I_{n}=\left(p, v_{1}, \cdots, v_{n-1}\right) \subset B P_{*}$. Our computations hold for all such $E_{*}(-)$ where on the coefficient rings, $\mu_{*}\left(I_{n}\right)=0$. For $p$ an odd prime, this class includes all $P(m), B(m), k(m)$, and $K(m), m \geqslant n$ as well as the usual mod $p$ homology $H_{*}(-)$; see [J-W] for these theories. For $p$ an odd prime, these are all nice multiplicative spectra by $[\mathrm{S}-\mathbb{Y}],[\mathbb{W} u]$, and $\left[\mathbb{M o}_{3}\right]$.

We show that $\left.E_{*} \underline{K(n)}\right)_{*}$ is a Hopf ring, i.e., each $E_{*} \underline{K(n)_{i}}$ is a (bi)com-

Communicated by N. Shimada, October 11, 1983.

* Research Institute for Mathematical Sciences, Kyoto University, Kyoto 606, Japan. Present Address: Mathematics Department, Johns Hopkins University, Baltimore, Maryland 21218, U.S.A. 
mutative Hopf algebra with conjugation and product $*$; and the ring structure of $K(n)^{*}(-)$ gives a pairing $\circ: E_{*} \underline{K(n)_{i}} \otimes_{E *} E_{*} \underline{K(n)}_{j} \rightarrow E_{*} \underline{K(n)}_{i+j}$. We make continual use of the techniques in our joint work with Ravenel on Hopf rings: $\left[\mathbf{R}-\mathbf{W}_{1}\right],\left[\mathbf{R}-\mathbf{W}_{2}\right]$ and Section 8 of $[\mathbf{W}]$. We use these techniques and the bar spectral sequence with Hopf rings from [T-W] to compute $H_{*} \underline{K(n)_{*}}$. There are many non-zero differentials and the nontrivial algebra extension problems all drop to the first filtration. We then use Hopf rings to show that the Atiyah-Hirzebruch spectral sequence collapses, and to solve all extension problems.

As with all of our previous calculations, $E_{*} \underline{K(n)_{*}}$ is generated by few elements. In Section 1 we define elements $a_{(i)} \in E_{2 p^{i}} \underline{K(n)}_{1}, i<n, b_{(i)} \in E_{2 p^{i}} \underline{K(n)_{2}}$, and $e_{1} \in E_{1} K(n)_{1}$. For $I=\left(i_{0}, i_{1}, \cdots, i_{n-1}\right)$ and $J=\left(j_{0}, j_{1}, \cdots\right)$, nonnegative finite sequences with $i_{k}=0$ or 1 and $j_{k}<p^{n}$, we define

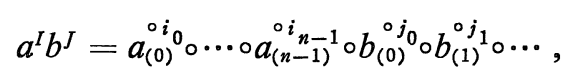

with the convention that $a^{I} b^{J}=[1]-[0]$ if $I$ and $J$ are all zeros. Let $E(x)$ be the exterior algebra with generator $x$ and $P(x)$ the polynomial algebra. Let $T P_{k}(x)$ be $P(x) / x^{p^{k}}$. Let $I(1)$ be the $I$ with all $i_{k}=1$.

Theorem 1. Let $E$ be a multiplicative BP module spectrum with $\mu_{*}\left(I_{n}\right)=0$. Let $p$ be an odd prime. As $E_{*}$ algebras

$$
E_{*} \underline{K(n)_{*}} \simeq \bigotimes_{j_{0}<p^{n}-1} E\left(a^{I} b^{J} \circ e_{1}\right) \underset{\substack{I \neq I(1) \\ \text { if } i_{0}=1, \\ \text { then } j_{0}<p^{n}-1}}{\bigotimes} T P_{\rho(I)}\left(a^{I} b^{J}\right) \bigotimes_{\substack{I=I(1) \\ j_{0}<p^{n}-1}} P\left(a^{I} b^{J}\right)
$$

where the tensor product is over $E_{*}$ and runs through all $I$ and $J$ (recall that $I$ and $J$ are defined to have all $i_{k}=0,1$, and $\left.j_{k}<p^{n}\right)$. The number $\rho(I)>0$ is the smallest $k$ with $i_{n-k}=0$.

The details of the Hopf ring relations are given in the text. For the prime 2, Theorem 1 holds as stated. Unfortunately the spectra of interest, e.g. $P(n)$, $K(n)$, may not be commutative ring spectra. Even in these cases the AHSS collapses giving $E_{*} \underline{K(n)_{*}}$ for $p=2$. Because of the possible lack of commutativity of the multiplications on $E$ and $K(n)$, this is not necessarily as algebras.

The author would like to thank David Johnson and Tony Bahri for their interest and acknowledge the influence of the joint work with Douglas Ravenel. Thanks are due to Professor Shimada and the RIMS, Kyoto University, for their hospitality during much of this project. We gratefully acknowledge 
the support of the Johns Hopkins University, the N.S.F., and the Ministry of Education in Japan.

In Section 1 we define the elements $a_{(i)}$ and $b_{(i)}$ and give the relations. Then we deduce Theorem 1 for $E_{*}(-)$ from Theorem 1 for $H_{*}(-)$. In Section 2 we do the homology computation. Comments on $p=2$ are at the end of each section.

\section{The Basics}

The results in this section, coupled with Theorem 1, complete the description of $E_{*} \underline{K(n)} *$ as a Hopf ring.

The spectrum $P(n)$ of $[\mathbf{J}-\mathbf{W}]$ has the property that $P(n)_{*} \simeq B P_{*} / I_{n}$. For $p$ odd it is the universal multiplicative $B P$ module spectrum with $\mu_{*}\left(I_{n}\right)=0$. This fact follows from the work of Würgler in [Wu] (see 6.8), where he computes $E^{*} B P$ and $E^{*} P(n)$. We crowd our basic facts into a long proposition.

Proposition 1.1. Let $p$ be an odd prime. We have elements $e_{1} \in E_{1} K(n)_{1}$, $a_{i} \in E_{2 i} \underline{K(n)_{1}}, i<p^{n}$, and $b_{i} \in E_{2 i} \underline{K(n)_{2}}$ such that: (Let $b_{(i)}=b_{p^{i}}$ and $\left.a_{(i)}=a_{p^{i}}.\right)$

(a) They are natural with respect to $E$.

(b) $e_{1} \circ-i s$ the homology suspension map.

(c) The coproduct is given by $a_{i} \rightarrow \sum a_{i-j} \otimes a_{j}, b_{i} \rightarrow \sum b_{i-j} \otimes b_{j}$.

(d) They are all permanent cycles in the AHSS for $P(n)_{*} \underline{K(n)_{*}}$.

(e) $e_{1} \circ e_{1}=b_{1}$.

(f) $a_{(i)} \circ a_{(j)}=-a_{(j)} \circ a_{(i)}$.

(g) $b_{(i)}{ }^{* p}=0$.

(h) $a_{(i)} * p=0, i<n-1$.

(i) $a_{(n-1)}{ }^{* p}=\mu_{*}\left(v_{n}\right) a_{(0)}-a_{(0)} \circ b_{(0)}^{\circ p^{n}-1}$.

(j) $\mu_{*}\left(v_{n}\right) e_{1}=b_{(0)}^{\circ p^{n}-1} \circ e_{1}$.

(k) $b_{(k)}^{o p^{n}}=\sum_{i=0}^{k} \mu_{*}\left(\nu_{n+i}^{p^{k-i}}\right) b_{(k-i)} \bmod *, k>0$.

(1) $b_{(k)}^{\stackrel{\circ}{n}}=\mu_{*}\left(v_{n}^{p^{k}}\right) b_{(k)}, k>0$, if $\mu_{*}\left(v_{n+i}\right)=0, i>0$.

Define $s(J)=\left(0, j_{0}, j_{1}, \cdots\right)$. If $i_{n-1}=0$, define $s(I)=\left(0, i_{0}, \cdots, i_{n-2}\right)$. Let $\Delta_{i}$ be the sequence with one in the $i^{\text {th }}$ place and zeros elsewhere. Define the length of $I$ to be $l(I)=\sum i_{k}$.

Proposition 1.2. Let $p$ be an odd prime and $E$ as usual.

(a) If $i_{n-1}=1$, then, in $E_{*} \underline{K(n)} *$

$$
\left(a^{I} b^{J}\right)^{* p}=(-1)^{l(I)} a^{s\left(I-\Delta_{n-1}\right)+\Delta_{0}} b^{s(J)+\left(p^{n}-1\right) \Delta_{0}}+(-1)^{l(I)-1} \mu_{*}\left(v_{n}\right) a^{s\left(I-\Delta_{n-1}\right)+\Delta_{0}} b^{s(J)} .
$$


(b) If $i_{n-1}=0$, then $\left(a^{I} b^{J}\right)^{* b}=0$ in $E_{*} \underline{K(n)_{*}}$.

(c) In Theorem 1 for $H_{*} \underline{K(n)} *$ we have, up to signs, an equality of sets (I and $J$ as usual)

$$
\begin{aligned}
& \left\{\left(a^{I} b^{J}\right)^{* p^{\rho(I)-1}} \mid I \neq I(1), \text { and if } i_{0}=1, \text { then } j_{0}<p^{n}-1\right\} \\
= & \left\{a^{I} b^{J} \mid i_{n-1}=0\right\} .
\end{aligned}
$$

Proof of Theorem 1 for general $E$. We assume Theorem 1 for $H_{*}(-)$. The $E^{2}$ term of the AHSS is $E_{*} \otimes_{z / p} H_{*} K(n)_{*}$ because $E_{*}$ must be a vector space over $Z / p$ by our assumptions about $E$. Because $P(n)$ is universal and the $a_{(i)}, b_{(i)}, e_{1}$, and the AHSS are all natural with respect to $E$, (1.1 (a)), the elements $a_{(i)}, b_{(i)}$ and $e_{1}$ are all permanent cycles in the AHSS for $E_{*} \underline{K(n)_{*}}$ by 1.1 (d). By Theorem 1 for homology, these elements generate the entire $E^{2}$ term as a module over $E_{*}$ (using both $*$ and $\circ$ products). It follows that the AHSS collapses. All of the extension problems are solved by 1.1 and 1.2; and the number $\rho(I)$ does not change.

Corollary 1.3. $E_{*} \underline{K(n)} *$ is a Hopf ring.

Proof. We have just proven that $E_{*} \underline{K(n)_{*}}$ is free over $E_{*}$. This implies a Künneth isomorphism for these spaces, so the coproduct can be defined. That was the only structure missing.

Proof of 1.1. We define our elements for $P(n)$ first and then use the universality of $P(n)$ to define them for all $E$. In degrees less than $2 p^{n}-1$ we have

$$
\widetilde{P(n)}_{*} \underline{K(n)}_{1} \cong \tilde{H}_{*} K(Z / p, 1)
$$

because $K(n)_{1} \simeq K(Z / p, 1)$ for dimensions less than $2 p^{n}-1$ and they are connected; while $P(n) \simeq H$ in stable dimensions less than $2\left(p^{n}-1\right)$. The groups $H_{1} K(Z / p, 1)$ and $H_{2 i} K(Z / p, 1)$ are isomorphic to $Z / p$ with canonical generators.

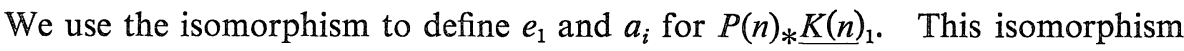
is natural because it is induced by maps $P(n) \rightarrow H$ and $K(n)_{1} \rightarrow K(Z / p, 1)$. The cohomology theory $K(n)^{*}(-)$ has a complex orientation, so we have a map $\boldsymbol{C} P^{\infty} \rightarrow K(n)_{2}$. Because $P(n)$ is a $B P$ module spectrum, $P(n)_{*} \boldsymbol{C} P^{\infty}$ is $P(n)_{*}$ free on $\beta_{i} \in P(n)_{2 i} C P^{\infty}$ (see [A]). Use these elements and the complex orientation to define the $b_{i} \in P(n)_{2 i} \underline{K(n)_{2}}$.

Part (a). Follows from the definition.

Part (b). The element $e_{1}$ is induced from $S^{1} \rightarrow \underline{K(n)_{1}}$. 
Part (c). The coproduct on the $a_{i}$ is induced from $H_{*} K(Z / p, 1)$. For the $b_{i}$, it comes from $\mathbb{C} P^{\infty}$, but only if $E_{*} \underline{K(n)_{2}}$ is a coalgebra. For $E=H$ it is, and so Theorem 1 and Corollary 2.3 are true, and $E_{*} \underline{K(n)_{2}}$ is a coalgebra.

Part (d). The $a_{i}$ and $e_{1}$ must be permanent cycles for the same dimensional reasons which give us their definition. The $\beta_{i}$ are permanent cycles for $\mathbb{C} P^{\infty}$, so by naturality the $b_{i}$ are also.

Part (e). This is elementary.

Part (f). Same as 9.1, $\left[\mathbb{R}-\mathbb{W}_{2}\right]$.

Part (g). Again, we must prove this first for homology, then we have 1.3 and the same proof works for general $E_{*}(-)$. Multiplication by $p$ is zero on $K(n)^{*}(-)$ so $p: \underline{K(n)_{2}} \rightarrow \underline{K(n)_{2}}$ is homotopically trivial. Thus $0=p_{*}: E_{*} K(n)_{2}$ $\rightarrow E_{*} \underline{K(n)_{2}} . \quad$ As in 7.1 of $\left[\mathbb{R}-\mathbb{W}_{2}\right], 0=p_{*} b_{(i+1)}=F V b_{(i+1)}=F b_{(i)}=b_{(i)} * p$.

Part (h). Same as (g).

Part (i). The only definable, possibly nonzero, elements in $P(n)_{2 p^{n}} \underline{K(n)_{1}}$ are the three in the equation; use the AHSS and dimensional arguments. We must have a formula $\left(r_{i} \in Z / p\right)$

$$
a_{(n-1)}^{* p p}=r_{1} v_{n} a_{(0)}+r_{2} a_{(0)} \circ b_{(0)}^{\circ p^{n}-1} \circ\left[v_{n}\right],
$$

where we have added the periodicity operator, [v $v_{n}$, to show that $a_{(0)} \circ b_{(0)}^{\circ p^{n}-1}$ really lies in $P(n)_{*} \underline{K(n)}_{2 p^{n}-1}$. We need this in a moment. First we map

$$
P(n)_{*} \underline{K(n)_{1}} \rightarrow K(n)_{*} K(Z / p, 1) .
$$

This map takes $b_{(0)}$ to zero and the above formula to $a_{(n-1)} * p=v_{n} a_{(0)}$ (5.7 of

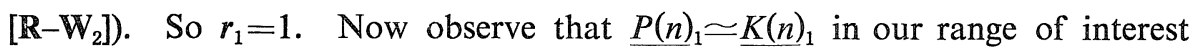
(actually in dimensions less than $2 p^{n+1}-1$ ). We map our formula to $P(n)_{*} P(n)$ by stabilization. By (b) this is just multiplication by $e_{1}$ an infinite number of times, remembering (e). Stabilizing kills $*$ products, so our formula becomes

$$
0=v_{n} \operatorname{im}\left(a_{(0)}\right)+r_{2} \operatorname{im}\left(a_{(0)}\right) v_{n} .
$$

Because $I_{n}$ is zero on both left and right, $v_{n}$ is the same on both left and right, so this is $0=v_{n}\left(1+r_{2}\right) \operatorname{im}\left(a_{(0)}\right)$. Both Yagita [Y] and Würgler [Wu] have computed $P(n)_{*} P(n)$ as

$$
P(n)_{*} B P \otimes_{P(n)_{*}} E\left(\operatorname{im} a_{(0)}, \cdots, \operatorname{im} a_{(n-1)}\right) .
$$


This is $P(n)_{*}$ free so $\left(1+r_{2}\right)$ im $a_{(0)}=0$, and $r_{2}=-1$. Note that in order to solve this problem precisely in homology it is necessary for us to do the general case first.

Part (j). The proof is similar to that for (i). These are the only two elements in this degree and they are equal after stabilization.

Part (k). From $\left[\mathbf{R}-\mathbf{W}_{1}\right], 3.11$, we have $[p](x)=\sum_{i \geqslant 0}^{F} v_{n+i} x^{p^{n+i}}$ for $P(n)$, and $[p](x)=v_{n} x^{p^{n}}$ for $K(n)$. We apply the main relation of $\left[\mathbf{R}-\mathbf{W}_{1}\right], 3.8$,

$$
\begin{aligned}
& b(s)^{\circ} p^{n} \circ\left[v_{n}\right]=[p]_{[F]_{K(n)}}\left(b_{(s)}\right)=b\left([p]_{F_{P(n)}}(s)\right) \\
= & b\left(\sum^{F} v_{n+i} s^{p^{n+i}}\right)=+_{[F]_{K(n)}} b\left(v_{n+i} s^{p^{n+i}}\right) .
\end{aligned}
$$

Modulo $*$ products, using the definition $b(s)=\sum b_{i} s^{i}$, the coefficient of $s^{p^{n+k}}$ gives $(\mathrm{k})$ as in 3.12 of $\left[\mathbf{R}-\mathbf{W}_{1}\right]$.

Part (1). It is unnecessary to go modulo $*$ products to prove this case of (k).

Proof of 1.2. We use the distributive law for Hopf rings, as in 7.1 of $\left[\mathbf{R}-\mathbf{W}_{2}\right]$.

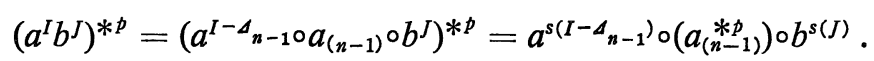

The rest follows from 1.1 (i) and (f). Part (b) is the same using (g) or (h) of 1.1. Part (c) follows from iterating (a) for $E=H$.

Modification for $p=2$.

Many things go through as stated for $p=2$. The elements $e_{1}, a_{i}$, and $b_{i}$ are all defined the same. However, to give the coproduct (part (c) of 1.1) it would be better to incorporate $e_{1}$ into the $a_{i}$, just as one would do for $H_{*} B Z / p$; i.e., let $a_{i} \in H_{i} \underline{K(n)_{1}}$ (then $a_{1}=e_{1}$ ). Part (f) implies $a_{(i)} \circ a_{(i)}=0$ for $p$ odd, but not for $p=2$. In fact, it is not zero for $p=2$.

The main problem for $p=2$ arises from the possible lack of commutativity for $E$ of interest. This lack of commutativity also shows up in $\underline{K(n)}_{*}$. Since $K(n)_{*}$ is an $\Omega$-spectrum there is no problem with the $*$ product from it. $E$, however, can mess up the $*$ product if $E$ is not commutative. For the $E$ of interest, $E=P(n), k(n)$, etc., we show that o product commutativity holds. From Würgler's work [Wu], we can still use the universality of $P(n)$. We show that for $p=2, a_{(i)} \circ a_{(j)}=a_{(j)} \circ a_{(i)}$ and $a_{(i)} \circ b_{(j)}=b_{(j)} \circ a_{(i)}$. Würgler computes the home of the obstruction to commutativity as $(\neq 1)$ 


$$
E_{E^{*}}\left(Q_{0}, Q_{1}, \cdots, Q_{n-1}\right) \otimes E_{E^{*}}\left(Q_{0}^{\prime}, Q_{1}^{\prime}, \cdots, Q_{n-1}^{\prime}\right),
$$

the primitives of $E^{*}(P(n) \wedge P(n))$. For $E=P(n)$, the $Q_{k}$ cover the Milnor primitive in mod 2 cohomology. In $P(n)_{*} \underline{P(n)_{2}} \simeq P(n)_{*} \underline{K(n)_{2}}$ (in our range), the obstruction for noncommutativity of $a_{(i)} \circ a_{(j)}$ factors through negative degrees, which are zero. For $a_{(i)} \circ b_{(j)}$, we see that $P(n)_{*} C P^{\infty}$ is even degree, so the $Q_{k}$ are all zero. This argument works for all $E$ with $E^{i}=0,0>i>-2\left(p^{n}-1\right)$.

There are no problems with mod 2 homology. Lack of commutativity destroys the nice Hopf algebra structure for arbitrary $E$ however.

In the stable object $K(n)_{*} K(n)$, see $[\mathbb{R}]$ and $[\mathbf{Y}]$, one of the defining relations is $v_{n} t_{k}^{p^{n}}=v_{n}^{p^{k}} t_{k}$. If we change generators we see that this relation occurs in the defining space unstably by 1.1 (1); a slightly unexpected result.

\section{§ 2. $H_{*} K(n)_{*}$}

We prove Theorem 1 by induction on degree using the bar spectral sequence with Hopf rings $[\mathbb{T}-\mathbf{W}]$. Let $K(n)_{i}^{\prime}$ be the zero component. Then

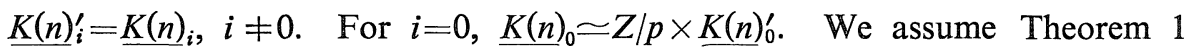
for $H_{i} \underline{K(n)_{*}}, i<k$. The bar spectral sequence determines $H_{i} \underline{K(n)_{*+1}^{\prime}}, i \leq k$, and so $H_{i} \underline{K(n)_{*}}, i \leqslant k$. This induction is implicit in this section. Let $\sigma$ be the suspension in the spectral sequence. We use the notation and basic facts of the spectral sequence from $\left[\mathbb{R}-\mathbb{W}_{2}\right]$. Let $m(J)$ be the smallest $k$ with $j_{k} \neq 0$. Define $m(I)$ similarly. Let $M(I)$ be the largest $k$ with $i_{k}=1$.

Theorem 2.1. Let $p$ be any prime. In the bar spectral sequence, $E_{* *}^{r}\left(H_{*} \underline{\left.K(n)_{*}\right)} \Rightarrow H_{*} \underline{K(n)_{*+1}^{\prime}}\right.$, we have

(a) $E_{* *}^{2} H_{*} \underline{K(n)} * \operatorname{Tor}_{* *}^{H * K(n) *}(Z / p, Z / p) \equiv H_{* *} H_{*} \underline{K(n)_{*}}$

$$
\simeq \bigotimes_{j_{0}<p^{n}-1} \Gamma\left(\sigma a^{I} b^{J} e_{1}\right) \underset{\begin{array}{c}
\text { if } i_{0}=1 \\
\text { then } j_{0}<p^{n}-1
\end{array}}{\otimes} E\left(\sigma a^{I} b^{J}\right) \bigotimes_{i_{n-1}=0} \Gamma\left(\phi a^{I} b^{J}\right),
$$

where the tensor products are indexed over I and J.

(b) In the Hopf ring pairing of the bar spectral sequence, [T-W], we have the following relations modulo decomposables and other terms mentioned in the proof.

For $J \neq 0$ and $k=m(J)$, consider

$$
\circ: H_{* *} H_{*} \underline{K(n)_{*-2}} \otimes H_{*} \underline{K(n)_{2}} \rightarrow H_{* *} H_{*} \underline{K(n)_{*}} .
$$

(i ) $\gamma_{p^{i}}\left(\sigma a^{I} b^{J-\Delta_{k}} e_{1}\right) \circ b_{(k+i)}=\gamma_{p^{i}}\left(\sigma a^{I} b^{J} e_{1}\right), j_{0}<p^{n}-1$. 
(ii) $r_{p^{i}}\left(\phi a^{I} b^{J-\Delta_{k}}\right) \circ b_{(k+i+1)}=\gamma_{p^{i}}\left(\phi a^{I} b^{J}\right), i_{n-1}=0$.

For $J=0$ and $m(I)=k$, consider

$$
\circ: H_{* *} H_{*} \underline{K(n)_{*-1}} \otimes H_{*} \underline{K(n)_{1}} \rightarrow H_{* *} H_{*} \underline{K(n)_{*}} \text {. }
$$

(iii) $\gamma_{p^{i}}\left(\sigma a^{I-\Delta_{k}} e_{1}\right) \circ a_{(k+i)}=(-i)^{l(I)} \gamma_{p^{i}}\left(\sigma a^{I} e_{1}\right), k+i<n$.

(iv) $\gamma_{p^{i}}\left(\phi a^{\left.I-\Delta_{k}\right) \circ a_{(k+i+1)}}=(-1)^{l(I)-1} \gamma_{p^{i}}\left(\phi a^{I}\right), i_{n-1}=0, k+i+1<n\right.$.

(c) Let $q=\eta(I)=\min \left\{j \mid i_{n-j}=1\right\}$ if $I \neq 0$, and $n+1$ if $I=0$. Let $I^{\prime}=I-\Delta_{n-q}$. The differentials are determined by:

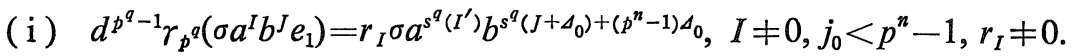

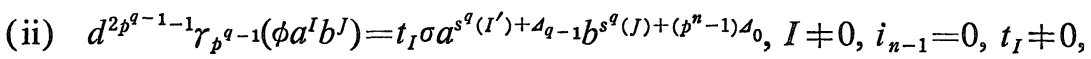

$$
=t_{0} \sigma b^{s^{q}(J)+\left(p^{n}-1\right) \Delta_{0}}, \quad I=0, t_{0} \neq 0 .
$$

(d) In $E_{* *}^{\infty}$ (modulo things mentioned in the proof)

$\gamma_{p^{i}}\left(\sigma a^{I} b^{J} e_{1}\right), j_{0}<p^{n}-1$, represents $a^{s^{i}(I)} b^{s^{i}\left(J+\Delta_{0}\right)} \quad$ where if $I \neq 0$, then $i<\eta(I)$

$\gamma_{p^{i}}\left(\phi a^{I} b^{J}\right), i_{n-1}=0$, represents $a^{s^{i+1}(I)+\Delta_{i} b^{s^{i+1}(J)}}$, where $i<\eta(I)-1$.

$\sigma a^{I} b^{J}, i_{0}=1 \Rightarrow j_{0}<p^{n}-1$, represents $(-1)^{l(I)} a^{I} b^{J} e_{1}$.

(e) As an algebra, $E_{* *}^{\infty}$ is

$$
\bigotimes_{j_{0}<p^{n}-1} E\left(a^{I} b^{J} e_{1}\right) \otimes T P_{1}\left(a^{I} b^{J}\right)
$$

where the tensor product is over I and J.

Proof of Theorem 1 for $H_{*}(-)$.

All that remains to do is to solve the algebra extension problems for 2.1 (e). This is done by 1.2. Now we have to check that we have Theorem 1 . The exterior part is okay. By 1.2, we know that if $a_{(0)} \circ b_{(0)}^{\circ p^{n}-1}$ divides $a^{I} b^{J}$, then $a^{I} b^{J}$ is not a generator; so our generators are correct. If $I=I(1)$, our generator is a polynomial generator by 1.2. Likewise 1.2 allows us to compute $\rho(I)$ for $I \neq I(1)$.

Proof of Theorem 2.1.

Part (a). See $\left[\mathbb{R}-\mathbb{W}_{2}\right], 6.6$ for basics. From the exterior part of Theorem 1 we get $\bigotimes_{j_{0}<p^{n}-1} \Gamma\left(\sigma a^{I} b^{J} e_{1}\right)$. From the generators of the other two terms we get $\bigotimes_{\text {if } i_{0}=1} E\left(\sigma a^{I} b^{J}\right)$. That concludes the polynomial algebra contribution. then $j_{0}<p^{n}-1$

From the truncated polynomial algebras we also have a transpotence contribution, which, by 1.2 (c), is our final term. 
Part (b). We prove these formulas for $i=0$. The $i>0$ cases follow by applying the iterated Verschiebung, $V^{i}$, see Section 7 of $\left[\mathbb{R}_{R}-\mathbb{W}_{2}\right]$.

For (i), we have easily;

$$
\sigma a^{I} b^{J-\Delta_{k}} e_{1} \circ b_{(k)}=\sigma a^{I} b^{J} e_{1} .
$$

On part (ii) we must be careful. For $k=0$ we have precisely

$$
\begin{aligned}
\left(\phi a^{I} b^{\left.J-\Delta_{0}\right) \circ b_{(1)}}\right. & =\left[\left(a^{I} b^{J-\Delta_{0}}\right)^{*-1} \mid a^{I} b^{J-\Delta_{0}}\right] \circ b_{(1)} \\
& =\left(a^{I} b^{J-\Delta_{0}} b_{(0)}\right)^{* p-1} \mid a^{I} b^{J-\Delta_{0}} b_{(0)}=\phi a^{I} b^{J} .
\end{aligned}
$$

If $k>0$ we replace $b_{(1)}$ with $b_{(k+1)}$ and $b_{(0)}$ with $b_{(k)}$. But now we could have other terms in the coproduct. However, if $k>0, a^{I} b^{J}$ is a generator, and never a $p$-th power, and we would be left with more than $p$ terms, thus they never contribute to the transpotences for elements with $k>0$. They may interfere with the $k=0, j_{0}=p^{n}-1$, cases, but we have solved them already and can mod them out at this stage. The (iii) and (iv) cases are now straightforward.

Part (c). Intuitively, all differentials follow from Hopf rings and $a_{(n)} \rightarrow$ $\sigma b^{\left(p^{n}-1\right) \Delta_{0}}$. Of course there is no $a_{(n)}$, and this is precisely why Miore complications arise because the differential $a_{(i)} \circ a_{(n)} \rightarrow \sigma a_{(i)} \circ b^{\left(p^{n}-1\right) \Delta_{0}}$ is shorter than the first one. This set, however, does generate all differentials. We collect the elementary differentials in a lemma whose proof we delay until after the general case is derived from them. Recall that since the target of a differential must be a primitive, and all of our generators above filtration one are even, the only possible targets are the exterior generators of part (a). Thus our only possible differentials are $d^{p^{i}-1}$ and $d^{2 p^{i}-1}$ on $\gamma_{p^{i}}$ of a suspension and a transpotence respectively.

$$
\begin{aligned}
& \text { Lemma。 (i ) } d^{p^{i}-1} \gamma_{p^{i}}\left(\sigma e_{1}\right)=0 \text { 。 } \\
& \text { (ii) } d^{p^{j}-1} \gamma_{p} j\left(\sigma a_{(k)} e_{1}\right)=0, \quad j<n-k, k<n \text {, } \\
& =r_{k} \sigma b^{\Delta_{n-k}+\left(p^{n}-1\right) \Delta_{0}}, \quad j=n-k, k<n, r_{k} \neq 0 . \\
& \text { (iii) } d^{2 p^{j}-1} \gamma_{p^{j}}\left(\phi a_{(k)}\right)=0, j<n-k-1, k<n-1 \text {, } \\
& =q_{k} \sigma a_{(n-k-1)} b^{\left(p^{n}-1\right) \Delta_{0}}, \quad j=n-k-1, k<n-1, q_{k} \neq 0 . \\
& \text { (iv) } d^{2 p^{i}-1} \gamma_{p^{i}}\left(\phi a^{I=0}\right)=0, \quad i<n \\
& =r \sigma b_{(0)}^{\circ p^{n}-1}, \quad i=n, r \neq 0 \text {. }
\end{aligned}
$$

We now prove part (c) from these differentials. We use from [T-W], the fact that $d^{j}(x \circ y)=d^{j}(x) \circ y$ our pairings in part (b). First we show by induction that if $I=0, j_{0}<p^{n}-1, J \neq 0$ 


$$
\begin{aligned}
& d^{p^{i}-1} \gamma_{p^{i}}\left(\sigma b^{J} e_{1}\right)=d^{p^{i}-1}\left(\gamma_{p^{i}}\left(\sigma b^{J-\Delta_{k}} e_{1}\right) \circ b_{(k+i)}\right) \\
= & d^{p^{i}-1}\left(\gamma_{p^{i}}\left(\sigma b^{J-\Delta_{k}} e_{1}\right)\right) \circ b_{(k+i)}=0 .
\end{aligned}
$$

Next, if $I \neq 0, J \neq 0$, and $j_{0}<p^{n}-1$ we compute, by induction on the length of $J$,

$$
\begin{aligned}
& d^{p^{i}-1} \gamma_{p^{i}}\left(\sigma a^{I} b^{J} e_{1}\right)=d^{p^{i}-1}\left(\gamma_{p^{i}}\left(\sigma a^{I} b^{J-\Delta_{k}} e_{1}\right) \circ b_{(k+i)}\right) \\
= & d^{p^{i}-1}\left(\gamma_{p^{i}}\left(\sigma a^{I} b^{J-\Delta_{k}} e_{1}\right)\right) \circ b_{(k+i)}=0 \quad \text { if } \quad i<q,
\end{aligned}
$$

and if $i=q$ it is equal to

$$
\begin{aligned}
& r_{I} \sigma a^{s^{q}\left(I^{\prime}\right)} b^{s^{q}\left(J-\Delta_{k}+\Delta_{0}\right)+\left(p^{n}-1\right) \Delta_{00}} b_{(k+q)} \\
= & r_{I} \sigma a^{s^{q}\left(I^{\prime}\right)} b^{s^{q}\left(J+\Delta_{0}\right)+\left(p^{n}-1\right) \Delta_{0} .} .
\end{aligned}
$$

If $J=0$ we do a similar induction on $l(I)$. We reduce to the lemma to prove (c) (i) using (b) (iii), also seeing that lower $\gamma_{p}{ }^{i}$ are cycles. For the (c) (ii) case we start with the lemma and use induction first on $l(I)$ and then $l(J)$ along with (b) (ii) and (iv). Again this also shows the differentials are zero for $r_{p}, i<q-1$.

We must show that this determines all of the differentials. In $\Gamma(x) \otimes E(y)$, if $d\left(r_{p^{i}}(x)\right)=y$, then the homology is $\bigotimes_{j<i} T P_{1}\left(\gamma_{p^{j}}(x)\right)$. In our case, since the targets are all distinct and the remaining $\gamma_{p}{ }^{j}(x)$ are all cycles, the spectral sequence must collapse after $E_{* *}^{2 p^{n}}$.

We prove the lemma. Part (i) follows by comparison with

$$
\Gamma\left(\sigma e_{1}\right) \cong H_{* *} H_{*} S^{1} \Rightarrow H_{*} C P^{\infty} .
$$

For the proof of part (d) note that this makes the identification for $b_{(i)}$.

For the part (ii), if $j<n-k$, we see that

$$
-r_{p}^{j}\left(\sigma a_{(k)} e_{1}\right)=r_{p^{j}}\left(\sigma e_{1}\right) \circ a_{(k+j)},
$$

and it follows from part (i). If $j=n-k$. Assume there is no differential, then

$$
\begin{aligned}
V\left(\gamma_{p^{n-k}}\left(\sigma a_{(k)} e_{1}\right)\right) & =\gamma_{p^{n-k-1}}\left(\sigma a_{(k)} e_{1}\right) \\
& =-\gamma_{p^{n-k-1}}\left(\sigma e_{1}\right) \circ a_{(n-1)} .
\end{aligned}
$$

We have just shown that this is a permanent cycle (which cannot be in the image of a differential) which is represented by $-b_{(n-k-1)} \circ a_{(n-1)}$. The $p$-th power of this is

$$
\left(-b_{(n-k-1)} \circ a_{(n-1)}\right)^{* p}=-b_{(n-k)} \circ\left(a_{(n-1)}^{* p}\right)=b_{(n-k)} \circ a_{(0)} \circ b_{(0)}^{\circ p^{n}-1}
$$

by 1.1 . We see soon that this is represented by $\gamma_{p}^{\circ}\left(\sigma a_{(0)} \circ b_{(n-k)} \circ b_{(0)}^{\circ p^{n}-2} \circ e_{1}\right)$ which is of even degree of filtration one so it survives nontrivially to $E_{* *}^{\infty}$. We 
know that $p_{*}: H_{*} \underline{K(n)_{*}} \rightarrow H_{*} \underline{K(n)} *$ must be zero because $K(n)_{*}(X)$ is a $Z / p$ vector space. But $p_{*}=F V$, see 7.1 of $\left[\mathbb{R}-\mathbb{W}_{2}\right]$. Thus we must have $d^{p^{n-k-1}}\left(\gamma_{p^{n-k}}\left(\sigma a_{(k)} e_{1}\right)\right) \neq 0$. We must discover the target. We know, $1.1(\mathrm{j})$, that $b_{(0)}^{\circ p^{n}-1} \circ e_{1}=\sigma b_{(0)}^{\circ p^{n}-1}=0$, so in 2.1 (a), all of the $\sigma a^{I} b^{J}$ with $j_{0}=p^{n}-1$, must be hit by differentials. We have already shown a one to one correspondence between such terms and $\Gamma(x)$ terms in (a) where $x \neq \sigma a^{I} b^{J} e_{1}, I=0$. In this last case we know they are permanent cycles anyway. The conclusion: the set of $\sigma a^{I} b^{J}$ with $j_{0}=p^{n}-1, i_{0} \neq 1$ are precisely the primitives which are targets of differentials! For the differential in question now, the degree $\sigma b^{\Delta_{n-k}+\left(p^{n}-1\right) \Delta_{0}}$ is the correct degree. Furthermore there are no other $\sigma a^{I} b^{J}$ of this degree with $j_{0}=p^{n}-1, i_{0}=0$. We are finished. The proof of part (iii) and (iv) of the lemma is similar. First we identify $\gamma_{p}\left(\phi a^{I=0}\right)$ with $a_{(j)}$ by comparison with $B Z / p$. These are permanent cycles. Then $\gamma_{p^{j}}\left(\phi a_{(k)}\right)=\gamma_{p^{j}}\left(\phi a^{I=0}\right) \circ a_{(k+j+1)}$ which is $a_{(j)} \circ a_{(k+j+1)}$ and is a cycles for $j<n-k-1$. If $j=n-k-1$ we apply $p_{*}=F V$ to $\gamma_{p^{j}}\left(\phi a_{(k)}\right)$. As above, $V \gamma_{p^{j}}\left(\phi a_{(k)}\right)=\gamma_{p^{j-1}}\left(\phi a_{(k)}\right)$ which we have just identified with $a_{(n-k-2)} \circ a_{(n-1)}$. The $p$-th power of this is $a_{(0)} \circ a_{(n-k-1)} \circ b_{(0)}^{\circ p^{n}-1}$ which we know to be represented by $r_{p} \circ\left(\sigma a_{(0)} \circ a_{(n-k-1)} \circ b_{(0)}^{\circ p^{n}-2} \circ e_{1}\right)$ and since it is even degree in filtration one, is nonzero in $E_{* *}^{\infty}$. Again, we must have a differential and the only candidate is the one advertised.

Part (d). In the process of proving part (c) we located $b_{(i)}$ and $a_{(i)}$. The rest follows by induction using part (b). This induction was essentially carried out when we showed that they were all cycles in the proof of part (c).

Part (e). See the comments in part (c) and part (d).

Modifications for $p=2$. Everything is fine for $p=2$, we are just using unusual notation. We should incorporate $e_{1}$ in the $a_{i}$ so that the coproduct is accurately reflected. For $p=2, E(x) \simeq T P_{1}(x)$, and as algebras, $H_{* *} T P_{1}(x)$ $\simeq \Gamma(\sigma x) \simeq E(\sigma x) \otimes \Gamma(\phi x)$, so we could simplify 2.1 considerably if we only did the $p=2$ case.

\section{References}

[A] J.F. Adams, Lectures on generalized cohomology, Lecture Notes in Math., 99, Springer-Verlag, Berlin, (1969) 1-138.

[J-W] D.C. Johnson and W.S. Wilson, BP operations and Morava's extraordinary $K$ theories, Math. Z., 144 (1975), 55-75.

[MRW] H.R. Miller, D.C. Ravenel and W.S. Wilson, Periodic phenomena in the AdamsNovikov spectral sequence, Ann. of Math. (2), 106 (1977), 469-516. 
[ $\left.\mathrm{Mo}_{1}\right]$ J. Morava, Structure theorems for cobordism comodules, preprint.

$\left[\mathrm{Mo}_{2}\right] \longrightarrow$, Extensions of cobordism comodules, preprint.

$\left[\mathrm{Mo}_{3}\right] \longrightarrow$, A product for odd-primary bordism of manifolds with singularities, Topology, 18 (1979), 177-186.

[R] D.C. Ravenel, The structure of Morava stabilizer algebras, Invent. Math., 37 (1976), 109-120.

$\left[\mathrm{R}-\mathrm{W}_{1}\right]$ D.C. Ravenel and W.S. Wilson, The Hopf ring for complex cobordism, J. Pure Appl. Algebra, 9 (1977), 241-280.

[R- $\left.\mathrm{W}_{2}\right]$ - The Morava $K$-theories of Eilenberg-MacLane spaces and the ConnerFloyd conjecture, Amer. J. Math., 102 (1980), 691-748.

[S-Y] N. Shimada and N. Yagita, Multiplications in complex bordism theory with singularities, Publ. RIMS, Kyoto Univ., 12 (1976), 259-293.

[T-W] R.W. Thomason and W.S. Wilson, Hopf rings in the bar spectral sequence, Quart. J. Math., 31 (1980), 507-511.

[W] W.S. Wilson, Brown-Peterson homology, An introduction and sampler. CBMS Regional Conference Series in Mathematics, 48. Amer. Math. Soc., Providence, 1982.

[Wu] U. Würgler, On products in a family of cohomology theories associated to the invariant prime ideals of $\pi_{*}(B P)$, Comment. Math. Helv., 52 (1977), 457-481.

[Y] N. Yagita, A topological note on the Adams spectral sequence based on Morava's K-theory. Proc. Amer. Math. Soc., 72 (1978), 613-617. 